\title{
AS CAUSAS DO COMPORTAMENTO OSCILATÓRIO DE SISTEMAS ELETROQUÍMICOS DEVIDO A IMOBILIZAÇÃO DAS SUBSTÂNCIAS DIFERENTES ATRAVÉS DA POLIMERIZAÇÃO ELETROQUÍMICA DE COMBINAÇÕES HETEROCÍCLICAS.
}

\author{
$\underline{\text { V.V.Tkach }}^{1}$ e V.V. Nechyporuk. \\ Ucrânia, Chernivtsi, Rua Kotsyubyns'kogo 2, Universidade Nacional de Chernivtsi, 58012 \\ ${ }^{1}$ E-mail do contato: volodya@llanera.com,nightwatcher2401@gmail.com
}

O comportamento de sistemas de imobilização de substâncias diferentes através da eletropolimerização das combinações heterocíclicas foi investigado através da teoria da estabilidade linear e da análise de bifurcações. Criando o modelo matemático deste processo e analizando-o nós prevemos que as oscilações podem sobrevir se ocorrer a interação entre as partículas adsortas e também a oxidação anôdica dos oxidantes fortes (incluindo também o crescimento autocatalítico da cadeia).

\section{INTRODUÇÃO}

Os polímeros condutores são as combinações orgânicas que atraem mais e mais interesse pois eles mantêm as caraterísticas dos plásticos e a condutividade dos metais. Tendo em vista as boas caraterísticas, podem ser utilizados em sensores [1,2], recobrimentos de proteção [3] e aparelhos eletroquímicos [4,5], além da retenção de substâncias tóxicas [2]. utilizados são:

Alguns dos polímeros condutores mais

- os derivados de alquinos (poliacetileno)

- os derivados de combinações aromáticas (poli-p-fenileno, polianilina)

- os derivados de combinações heterocíclicas de 5 átomos no ciclo (polifurano, polipirrol, politiofeno e os derivados destes).

Embora eles possam ser sintetizados tanto por síntese química quanto pela eletroquímica, a eletropolimerização ganha mais e mais atenção pois os polímeros obtidos pela síntese eletroquímica (anôdica e raramente catôdica [6]) mantém maior capacidade de conduzir corrente e de se modificar para manter as caraterísticas precisas. É possível funcionalizar os polímeros condutores por 4 métodos.
-A eletropolimerização de monômeros substituídos (às vezes pode ser autoinibidora e pode requerer a proteção dos grupos funcionais)-

-A eletrocopolimerização de dois monômeros para o copolímero manter as caraterísticas de ambos homopolímeros (não pode ser usada para todos os pares de monômeros). -A modificação do polímero já sintetisado. -A imobilização dos modificadores pela eletropolimerização na sua presença.

Ao sintetisar o polímero para as finaldades analíticas ou para retenção de substâncias tóxicas, se deve controlar as suas carateristicas para a implementação de funções precisas.Quando a substância é imobilizada pela polimerização eletroquímica, ela toma parte na reação. O polímero então ganha as caraterísticas novas. Porém às vezes as instabilidades eletroquímicas (oscilatórias ou monotônicas) podem ocorrer nesses sistemas $[3,6,7,8,9]$. Para poder explicar os fatos experimentais do seu aparecimento, sua influência no polímero formado e os procedimentos para evitá-las é preciso construir o modelo matemático deste processo para poder determinar o mecanismo mais provável associado. 


\section{A IMOBILIZAÇÃO DAS SUBSTÂNCIAS DIFERENTES PELA POLIMERIZAÇÃO ELETROQUÍMICA E O SEU MODELO MATEMÁTICO}

Para descrever a cinética da eletropolimerização, é melhor usar o mecanismo modificado de Diaz, segundo o qual a polimerização inclúi a oxidação do monômero formando o radical-cátion, as reações do crescimento da cadeia (a recombinação ou substituição eletrofílica) que formam o dímero, a oxidação dele (formação do centro do crescimento), a formação do trímero e a crescimento da cadeia da maneira já descoberta. O dopante participa no processo da polimerização estabilizando o radical-cátion, deminuindo o potencial da eletropolimerização e (caso que seja um dos oxidantes fortes) pode transferir o centro ativo à molécula do monômero livre.

A imobilização das substâncias pode resultar na formação dos complexos, nas formações das ligações covalente e sua incorporação nas cadeias polimericas e também no aprisionamento das moléculas durante a formação da rede polimérica[1].

A polimerização eletroquímica das combinações heterocíclicas pode ser realizada galvanostaticamente, potenciostaticamente e potenciodinamicamente, porém a polimerização potenciostática se usa na maioria dos casos. Por isso analisamos matematicamente o caso da polimerização nas condições do potencial constante.

Para criar o modelo matemático deste sistema pode-se introduzir as três variáveis:

$\mathrm{c}_{\mathrm{h}}$ - a concentração do monômero heterocíclico na câmada da difusão

$\theta_{\mathrm{h}}-\mathrm{o}$ grau do recobrimento da superfície com o monômero

$\mathrm{c}_{\mathrm{m}}$ - a concentração da substância que se imobiliza pela eletropolimerização.

Suposições: Para simplificar o modelo matemático deste processo supomos: -que o liquido está em constante movimento (não se considerando asssim a influência do fluxo da convecção); -que o eletrólito suporte (que contém o dopante) está presente em excesso, pois pode-se assim não se considerar a influência do fluxo da migração e a diminuição na concentração do dopante; -e que o polímero não sai da superfície por ser liofóbico. A distribuição de concentração do monômero na câmada da difusão é suposta ser linear e a espessura da camada constante e igual a $\delta$.

O monômero na câmada pre-superficial. O monômero entre na camada pre-superficial por difusão e também pela desorção e sai dela sendo adsorvido na superfície do eletrodo. A equação do balanço da concentração pre-superficial do monômero pode-se escrever como

$$
\frac{d c_{h}}{d t}=\frac{2}{\delta}\left(r_{-1}-r_{1}+\frac{D}{\delta}\left(c_{h, b u l k}-c_{h}\right)\right)
$$

Sendo que D significa o coeficiente da difusão, $\mathrm{c}_{\mathrm{h} \text {,buk }}$ significa a concentração do monômero no interior da solução, $r_{1}$ e $r_{-1}$ são as velocidades da adsorção e desorção.

O monômero na superfície. O monômero chega na superfície pela adsorção e sai dela pela desorção. Ele também é consumido, formando o polímero. A equação do balanço do grau do recobrimento da superfície com o monômero será descrita como.

$$
\frac{d \Theta_{h}}{d t}=\tilde{A}_{h, \max }\left(r_{1}-r_{-1}-r_{2}\right)
$$

Em que $r_{2}$ é a velocidade da polimerização eletroquímica e $\mathscr{A}_{h, \max }$ é a concentração superficial máxima do monômero.

A substância que se imobiliza. Chega à camada pre-superficial através da difusão do interior da solução e sai dela participando na reação da polimerização eletroquimica. A equação do balanço então pode-se descrever como

$$
\frac{d c_{m}}{d t}=\frac{2}{\delta}\left(\frac{D}{\delta}\left(c_{m, \text { buli }}-c_{m}\right)-r_{2}\right)
$$

Sendo que $\mathrm{c}_{\mathrm{h}, \text { bulk }}$ significa a concentração da substância que se imobiliza no interior da solução.

Para investigar o comportamento eletroquímico deste processo é preciso resolver as equações no sistema.

$$
\left\{\begin{array}{l}
\frac{d c_{h}}{d t}=\frac{2}{\delta}\left(r_{-1}-r_{1}+\frac{D}{\delta}\left(c_{h, b u l k}-c_{h}\right)\right) \\
\frac{d \Theta_{h}}{d t}=\tilde{A}_{h, \text { max }}\left(r_{1}-r_{-1}-r_{2}\right) \\
\frac{d c_{m}}{d t}=\frac{2}{\delta}\left(\frac{D}{\delta}\left(c_{m, \text { bulk }}-c_{m}\right)-r_{2}\right)
\end{array}\right.
$$


A velocidade da eletropolimerização pode se descrever como

$$
r_{2}=k_{2} \theta_{h}^{x} c_{m}^{y} \exp \left(\frac{z F}{R T} \phi_{0}\right)
$$

Sendo que $\mathrm{k}_{2}$ é a constante da reação, $\mathrm{x}$ e y são as ordens da reação do monômero e da substância que está se imobilizando, z é a quantidade dos eletrons transferidos, F é a constante de Faraday, R é a constante universal da gases, $\mathrm{T}$ é a temperatura absoluta do vaso e $\varphi_{0}$ significa o salto do potencial relativemente ao potencial da carga zero.

As velocidades da adsorção e desorção podem-se calcular como

$$
\begin{gathered}
r_{1}=k_{1} \exp \left(-\frac{\left(K_{0}-K_{1}\right) \phi_{0}^{2}+2 \mathrm{~K}_{2} \phi_{0} \phi_{1}}{2 R T \tilde{A}_{\max 1}} \gamma\right) \exp \left(a\left(\theta_{h}\right) c_{h}\left(1-\theta_{h}\right)\right. \\
r_{-1}=k_{-1} \exp \left(\frac{\left(K_{0}-K_{1}\right) \phi_{0}^{2}+2 \mathrm{~K}_{2} \phi_{0} \phi_{2}}{2 R T \tilde{A}_{\max 1}}(1-\gamma) \exp \left(-a \theta_{h}\right) \theta_{h}\right.
\end{gathered}
$$

Em que $\mathrm{k}_{1}$ e $\mathrm{k}_{-1}$ são as constantes da adsorção e desorção do monômero, $\mathrm{K}_{1}$ e $\mathrm{K}_{0}$ significam as capacidades elétricas das partes da câmada pre-superficial que se referem à superficie coberta pelo monômero e à livre, $\varphi_{0}$ significa o salto do

potencial relativamente ao potencial de carga zero relativamente à parte da superfície coberta pelo monômero.

\section{A ANÁLISE DA ESTABILIDADE LINEAR}

O comportamento eletroquímico destes sistemas é analisado observando pela teoria da estabilidade linear. A matriz funcional de Jacobi podese descrever como
Os membros desta matriz estão calculados para o estado estacionário e são dados por

$$
\begin{gathered}
a_{11}=\frac{\partial F_{1}}{\partial c_{h}}=\frac{2}{\delta}\left(-\frac{\partial r_{1}}{\partial c}-\frac{D}{\delta}\right) \quad a_{12}=\frac{\partial F_{1}}{\partial \theta_{h}}=\frac{2}{\delta}\left(-\frac{\partial r_{1}}{\partial \theta_{h}}+\frac{\partial r_{-1}}{\partial \theta_{h}}\right) \\
a_{13}=\frac{\partial F_{1}}{\partial c_{m}}=0 \\
a_{21}=\frac{\partial F_{2}}{\partial c_{0}}=\Gamma_{\max 1}^{-1} \frac{\partial r_{1}}{\partial c_{h}} \\
a_{22}=\frac{\partial F_{2}}{\partial \theta_{h}}=\Gamma_{\max 1}^{-1}\left(\frac{\partial r_{1}}{\partial \theta_{h}}-\frac{\partial r_{-1}}{\partial \theta_{h}}-\frac{\partial r_{2,}}{\partial \theta_{h}}\right) \\
a_{23}=\frac{\partial F_{2}}{\partial c_{m}}=\Gamma_{\max 1}^{-1}\left(-\frac{\partial r_{2,}}{\partial c_{m}}\right) \quad a_{31}=\frac{\partial F_{3}}{\partial c_{h}}=0 \\
a_{32}=\frac{\partial F_{3}}{\partial \theta_{h}}=-\frac{\partial r_{2,}}{\partial \theta_{h}} \quad a_{33}=\frac{\partial F_{3}}{\partial c_{m}}=\frac{2}{\delta}\left(-\frac{\partial r_{2}}{\partial c_{m}}-\frac{D}{\delta}\right)
\end{gathered}
$$

As oscilações através da implementação das condições da bifurcação de Hopf podem sobrevir se os elementos da diagonal principal contiverem as parcelas positivas (as quais são responsáveis pela conexão da volta positiva). Os elementos da diagonal principal da matriz podem ser calculados por

$$
a_{11}=\frac{\partial F_{1}}{\partial c_{h}}=\frac{2}{\delta}\left(-\frac{\partial r_{1}}{\partial c}-\frac{D}{\delta}\right)
$$

$$
\begin{aligned}
J=\left(\begin{array}{lll}
a_{11} & a_{12} & a_{13} \\
a_{21} & a_{22} & a_{23} \\
a_{31} & a_{32} & a_{33}
\end{array}\right) & a_{33}=\frac{\partial F_{3}}{\partial c_{m}}=\frac{2}{\delta}\left(-\frac{\partial r_{2}}{\partial c_{m}}-\frac{D}{\delta}\right) \\
& a_{22}=\frac{\partial F_{2}}{\partial \theta_{h}}=\Gamma_{\max }^{-1}\left(\frac{\partial r_{1}}{\partial \theta_{h}}-\frac{\partial r_{-1}}{\partial \theta_{h}}-\frac{\partial r_{2}}{\partial \theta_{h}}\right)= \\
& =\left(a r_{1}-\frac{r_{1}}{1-\theta_{h}}-\frac{\left(K_{0}-K_{1}\right) \varphi_{0}+K_{1} \varphi_{1}}{R T \Gamma_{\max 1}} r_{1} \frac{\partial \varphi_{0}}{\partial \theta_{h}}+a r_{-1}-\frac{w_{-1}}{\theta_{h}}-\frac{\left.\left(K_{0}-K_{1}\right) \varphi_{0}+K_{1} \varphi_{1}\right)}{R T \Gamma_{\max 1}}\right) \\
& *\left((1-\gamma) r_{-1} \frac{\partial \varphi_{0}}{\partial \theta_{h}}-x \frac{r_{2}}{\theta_{h}^{x}} \theta_{h}^{x-1}-\alpha \frac{z F}{R T} w_{2} \frac{\left(K_{0}-K_{1}\right) \varphi_{0}+K_{1} \varphi_{1}}{K_{0}\left(1-\theta_{h}\right)+K_{1} \theta}\right)
\end{aligned}
$$


A primeira causa das oscilações neste processo pode ser a interação atrativa entre as partículas adsorvidas. Isso explica as oscilações no caso de $\boldsymbol{a}_{1}+\boldsymbol{a}_{-1}>0$. Isso confirma a presença da instabilidade superficial, suposta em [6].

Esta interação é suportada:

-pela interação coulômbica entre o radical-cátion e a molécula do monômero (que se segue pela substituição eletrofílica)

-pela interação "spin-spin” entre os dois radical-cátions (que se segue pela recombinação).

Mais um fator que pode levar às oscilações neste processo podem ser a oxidação anôdica dos redutores fortes que se formam durante o processo da polimerização.

Isso pode explicar as oscilações no caso de

$$
\alpha \frac{z F}{R T} w_{2} \frac{\left(K_{0}-K_{1}\right) \varphi_{0}+K_{1} \varphi_{1}}{K_{0}\left(1-\theta_{h}-\theta_{h}\right)+K_{1} \theta}<0
$$

Isso sucede na condição de $\varphi_{0}<0$ (o que sobrevém durante a oxidação anódica dos redutores fortes). Como a partícula que se oxida no próximo passo da polimerização tem o sistema conjugado mais longo que o anterior, ele se oxida então mais rápido. Isso se manifesta no crescimento autocatalítico da cadeia.

Este fator explica também a diferença na amplitude das oscilações para as condições diferentes da síntese.

A instabilidade monotônica pode sobrevir nas condições de $\operatorname{Tr} \mathrm{J}<0$, Det $=0$ em que $\operatorname{Tr} \mathrm{J}=\mathrm{a}_{11}+\mathrm{a}_{22}+\mathrm{a}_{33}$ e Det $\mathbf{J}=$

$$
\left|\begin{array}{lll}
a_{11} & a_{12} & a_{13} \\
a_{21} & a_{22} & a_{23} \\
a_{31} & a_{32} & a_{33}
\end{array}\right|
$$

A primeira condição se satisfaz na maioria dos casos e a principal será a segunda.Ela pode ser satisfeita por exemplo se no processo da eletropolimerização ocorrer o "ponto morto" em que a polimerização se detém.

\section{CONCLUSÕES}

Investigou-se o processo da imobilização das substâncias diferentes através da eletropolimerização eletroquímica das combinações heterocíclicas na presença delas.

Analizando o modelo matemático deste processo podemos prever que as oscilações sobrevêm devido a interação atrativa entre as partículas adsortas e também pela oxidação anôdica dos redutores fortes que sucede durante o processo da eletropolimerização.

A instabilidade monotônica é também possível neste processo e por exemplo pode suceder se no processo da polimerização eletroquímica ocorrer o "ponto morto".

\section{REFERÊNCIAS}

[1]. V.M. de Andrade, Tés. M. Eng. UFRS., Porto Alegre, 2006

[2]. M. Brito Sousa, É. J.Dallan, R. Bertazolli, Quím. Nova. 23(3) (2000)

[3]. K.R. Lemos Castagno, Tés. D. Sc. UFRS.,Porto Alegre, 2007

[4]. M. Ates. Int. J. Electroch. Sci. 4 (2009)

[5]. Pourabbas B.; Pilati F.; Abstracts 6th Nanoscience and Nanotechnology conference, Izmir, Turkey, 2010

[6]. Roncali J.; Chem. Rev., 92(1992), 711

[7]. Das I.;Agrawal N.R.; Ansari S.A.; Gupta S.K. Ind. J. Chem., 47A.(2008)

[8]. Tkach V.V; Nechyporuk V.V.; Hrynda Yu.M.; J. Math. Sci. Eng. 5 (2011) 4

[9]. V.Tkach; V. Nechyporuk.; Abstracts Second world congress in Nanomedicine proceedings", Antalya, Turkey, 2010 